\title{
Some new inverse-type Hilbert-Pachpatte inequalities
}

Zhao Changjian and Mihály Bencze 


\title{
SOME NEW INVERSE-TYPE HILBERT-PACHPATTE INEQUALITIES
}

\author{
ZHAO CHANGJIAN AND MIHÁLY BENCZE
}

Received 2 June, 2006

\begin{abstract}
Inverses of some new inequalities similar to the Hilbert-Pachpatte inequality are established.
\end{abstract}

1991 Mathematics Subject Classification: 26D15

Keywords: Hilbert inequality, Hölder integral inequality, Jensen inequality

\section{INTRODUCTION}

In recent years several authors $[2,3,5-10]$ have given considerable attention to the Hilbert inequalities and Hilbert type inequalities and their various generalizations. In particular, in 1988, B. G. Pachpatte [6] proved some new inequalities similar to Hilbert's inequality [4, p. 226], The main purpose of this paper is to establish their inverses.

\section{MAIN RESULTS}

Our main results are given in the following theorems.

Theorem 1. Let $0<p \leq 1,0<q \leq 1$ and $\left\{a_{m}\right\}$ and $\left\{b_{n}\right\}$ be two nonnegative sequences of real numbers defined for $m=1,2, \ldots, k$ and $n=1,2, \ldots, r$, where $k$ and $r$ are the natural numbers and define $A_{m}=\sum_{s=1}^{m} a_{s}$ and $B_{n}=\sum_{t=1}^{n} b_{t}$. Then

For the first author, the research is supported by Zhejiang Provincial Natural Science Foundation of China (Y605065), Foundation of the Education Department of Zhejiang Province of China (20050392), and the Academic Mainstay of Middle-age and Youth Foundation of Shandong Province of China (200203). 
for $3 n-2 m>0$

$$
\begin{aligned}
& \sum_{m=1}^{k} \sum_{n=1}^{r} \frac{A_{m}^{p} B_{n}^{q}}{3 n-2 m} \geq p q k^{-2} r^{3}\left(\sum_{m=1}^{k}(\right.\left.k-m+1)\left(a_{m} A_{m}^{p}\right)^{-\frac{11}{3}}\right)^{3} \\
& \times\left(\sum_{n=1}^{r}(r-n+1)\left(b_{n} B_{n}^{q-1}\right)^{-\frac{1}{2}}\right)^{-2}
\end{aligned}
$$

Proof. By using the following inequality (see [4, p. 39])

$$
r x^{r-1}(x-y) \leq x^{r}-y^{r}, \quad 0<r \leq 1,
$$

where $x$ and $y$ are positive real numbers, we obtain that

$$
\sum_{m=0}^{k-1} p A_{m+1}^{p-1}\left(A_{m+1}-A_{m}\right) \leq \sum_{m=0}^{k-1}\left(A_{m+1}^{p}-A_{m}^{p}\right),
$$

that is

$$
A_{k}^{p} \geq p \sum_{m=1}^{k} a_{m} A_{m}^{p-1} .
$$

From (2) and in view of Hölder's inequality [4, p. 24], we have

$$
\begin{gathered}
\frac{A_{m}^{p} B_{n}^{q}}{3 n-2 m} \geq p q(3 n-2 m)^{-1}\left(\sum_{s=1}^{m} a_{s} A_{s}^{p-1}\right)\left(\sum_{t=1}^{n} b_{t} B_{t}^{q-1}\right) \\
\geq p q(3 n-2 m)^{-1} n^{3} m^{-2}\left(\sum_{s=1}^{m}\left(a_{s} A_{s}^{p-1}\right)^{\frac{1}{3}}\right)^{3} \\
\times\left(\sum_{t=1}^{n}\left(b_{t} B_{t}^{q-1}\right)^{-\frac{1}{2}}\right)^{-2} .
\end{gathered}
$$

Using the following inequality [1, p. 15]

$$
m^{\frac{1}{h}} n^{\frac{1}{l}} \geq \frac{m}{h}+\frac{n}{l}
$$

where $m>0, n>0, h^{-1}+l^{-1}=1$, and $h<1$, we easily get that

$$
n^{3} m^{-2} \geq 3 n-2 m \text {. }
$$

Taking the sum on both sides of (3) over $n$ from 1 to $r$ first and then the sum over $m$ from 1 to $k$ and in view of inequality (5) and using again Hölder's inequality, we 
obtain

$$
\begin{gathered}
\sum_{m=1}^{k} \sum_{n=1}^{r} \frac{A_{m}^{p} B_{n}^{q}}{3 n-2 m} \geq p q\left(\sum_{m=1}^{k}\left(\sum_{s=1}^{m}\left(a_{s} A_{s}^{p-1}\right)^{\frac{1}{3}}\right)^{3}\right) \\
\times\left(\sum_{n=1}^{r}\left(\sum_{t=1}^{n}\left(b_{t} B_{t}^{q-1}\right)^{-\frac{1}{2}}\right)^{-2}\right) \\
\geq p q k^{-2} r^{3}\left(\sum_{m=1}^{k}\left(\sum_{s=1}^{m}\left(a_{s} A_{s}^{p-1}\right)^{\frac{1}{3}}\right)\right)^{3}\left(\sum_{n=1}^{r}\left(\sum_{t=1}^{n}\left(b_{t} B_{t}^{q-1}\right)^{-\frac{1}{2}}\right)\right)^{-2} \\
=p q k^{-2} r^{3}\left(\sum_{s=1}^{k}\left(a_{s} A_{s}^{p-1}\right)^{\frac{1}{3}}\left(\sum_{m=s}^{k} 1\right)\right)^{3}\left(\sum_{t=1}^{r}\left(b_{t} B_{t}^{q-1}\right)^{-\frac{1}{2}}\left(\sum_{n=t}^{r} 1\right)\right)^{-2} \\
=p q k^{-2} r^{3}\left(\sum_{s=1}^{k}(k-s+1)\left(a_{s} A_{s}^{p-1}\right)^{\frac{1}{3}}\right)^{3} \\
\times\left(\sum_{t=1}^{r}(r-t+1)\left(b_{t} B_{t}^{q-1}\right)^{-\frac{1}{2}}\right)^{-2} \\
=p q k^{-2} r^{3}\left(\sum_{m=1}^{k}(k-m+1)\left(a_{m} A_{m}^{p-1}\right)^{\frac{1}{3}}\right)^{3} \\
\times\left(\sum_{n=1}^{r}(r-n+1)\left(b_{n} B_{n}^{q-1}\right)^{-\frac{1}{2}}\right)^{-2},
\end{gathered}
$$

which completes the proof.

Remark 1. Inequality (1) is just an inverse of the following Inequality A, which was proved by Pachpatte [6]:

Inequality A.

$$
\begin{aligned}
\sum_{m=1}^{k} \sum_{n=1}^{r} \frac{A_{m}^{p} B_{n}^{q}}{m+n} \leq \frac{1}{2} p q(k r)^{\frac{1}{2}}\left(\sum_{m=1}^{k}(k-m\right. & \left.+1)\left(a_{m} A_{m}^{p-1}\right)^{2}\right)^{\frac{1}{2}} \\
& \times\left(\sum_{n=1}^{r}(r-n+1)\left(b_{n} B_{n}^{q-1}\right)^{2}\right)^{\frac{1}{2}} .
\end{aligned}
$$


Theorem 2. Let $\left\{a_{m}\right\},\left\{b_{n}\right\}, A_{m}, B_{n}$ be as defined in Theorem 1. Let $\left\{p_{m}\right\}$ and $\left\{q_{n}\right\}$ be two positive sequences for $m=1,2, \ldots, k$ and $n=1,2, \ldots, r$, where $k$ and $r$ are natural numbers and put $P_{m}=\sum_{s=1}^{m} p_{s}, Q_{n}=\sum_{t=1}^{n} q_{t}$. Let $\phi$ and $\psi$ be two real-valued nonnegative, concave, and supermultiplicative functions ${ }^{*}$ defined on $\mathbb{R}_{+}$. Then for $3 n-2 m>0$

$$
\begin{aligned}
\sum_{m=1}^{k} \sum_{n=1}^{r} \frac{\phi\left(A_{m}\right) \psi\left(B_{n}\right)}{3 n-2 m} \geq M^{\prime}(k, r) & \left(\sum_{m=1}^{k}\left(p_{m} \phi\left(\frac{a_{m}}{p_{m}}\right)\right)^{\frac{1}{3}}(k-m+1)\right)^{3} \\
\times & \left(\sum_{n=1}^{r}\left(q_{n} \psi\left(\frac{b_{n}}{q_{n}}\right)\right)^{-\frac{1}{2}}(r-n+1)\right)^{-2}
\end{aligned}
$$

where

$$
M^{\prime}(k, r)=\left(\sum_{m=1}^{k}\left(\frac{\phi\left(P_{m}\right)}{P_{m}}\right)^{-\frac{1}{2}}\right)^{-2}\left(\sum_{n=1}^{r}\left(\frac{\psi\left(Q_{n}\right)}{Q_{n}}\right)^{\frac{1}{3}}\right)^{3}
$$

Proof. From the hypotheses and by Jensen's inequality and Hölder's inequality, we obtain

$$
\begin{aligned}
\frac{\phi\left(A_{m}\right) \psi\left(B_{n}\right)}{3 n-2 m} \geq(3 n-2 m)^{-1} & \\
& \times \phi\left(P_{m}\right) \psi\left(Q_{n}\right) \phi\left(\frac{\sum_{s=1}^{m} p_{s} a_{s} / p_{s}}{\sum_{s=1}^{m} p_{s}}\right) \psi\left(\frac{\sum_{t=1}^{n} q_{t} b_{t} / q_{t}}{\sum_{t=1}^{n} q_{t}}\right) \\
\geq & (3 n-2 m)^{-1} \frac{\phi\left(P_{m}\right)}{P_{m}} \frac{\psi\left(Q_{n}\right)}{Q_{n}} \sum_{s=1}^{m} p_{s} \phi\left(\frac{a_{s}}{p_{s}}\right) \sum_{t=1}^{n} q_{t} \phi\left(\frac{b_{t}}{q_{t}}\right) \\
\geq & (3 n-2 m)^{-1} n^{3} m^{-2} \frac{\phi\left(P_{m}\right)}{P_{m}} \frac{\psi\left(Q_{n}\right)}{Q_{n}}\left(\sum_{s=1}^{m}\left(p_{s} \phi\left(\frac{a_{s}}{p_{s}}\right)\right)^{\frac{1}{3}}\right)^{3} \\
& \times\left(\sum_{t=1}^{n}\left(q_{t} \psi\left(\frac{b_{t}}{q_{t}}\right)\right)^{-\frac{1}{2}}\right)^{-2}
\end{aligned}
$$

${ }^{*} f$ is said to be a supermultiplicative function if $f(x y) \geq f(x) f(y)$ for $x, y \in \mathbb{R}_{+}:=[0,+\infty)$. 
Taking the sum over $n$ from 1 to $r$ first and then the sum over $m$ from 1 to $k$ and using (5), in view of Hölder's inequality, we have

$$
\begin{aligned}
& \sum_{m=1}^{k} \sum_{n=1}^{r} \frac{\phi\left(A_{m}\right) \psi\left(B_{n}\right)}{3 n-2 m} \geq \sum_{m=1}^{k}\left(\frac{\phi\left(P_{m}\right)}{P_{m}}\left(\sum_{s=1}^{m}\left(p_{s} \phi\left(\frac{a_{s}}{p_{s}}\right)\right)^{\frac{1}{3}}\right)^{3}\right) \\
& \times \sum_{n=1}^{r}\left(\frac{\psi\left(Q_{n}\right)}{Q_{n}}\left(\sum_{t=1}^{n}\left(q_{t} \psi\left(\frac{b_{t}}{q_{t}}\right)\right)^{-\frac{1}{2}}\right)^{-2}\right) \\
& \geq\left(\sum_{m=1}^{k}\left(\frac{\phi\left(\left(P_{m}\right)\right)}{P_{m}}\right)^{-\frac{1}{2}}\right)^{-2}\left(\sum_{m=1}^{k} \sum_{s=1}^{m} p_{s} \phi\left(\frac{a_{s}}{p_{s}}\right)^{\frac{1}{3}}\right)^{3} \\
& \times\left(\sum_{n=1}^{r}\left(\frac{\psi\left(Q_{n}\right)}{Q_{n}}\right)^{\frac{1}{3}}\right)^{3}\left(\sum_{n=1}^{r} \sum_{t=1}^{n} q_{t} \psi\left(\frac{b_{t}}{q_{t}}\right)^{-\frac{1}{2}}\right)^{-2} \\
& =M^{\prime}(k, r)\left(\sum_{s=1}^{k}\left(p_{s} \phi\left(\frac{a_{s}}{p_{s}}\right)\right)^{\frac{1}{3}}\left(\sum_{m=s}^{k} 1\right)\right)^{3} \\
& \times\left(\sum_{t=1}^{r}\left(q_{t} \psi\left(\frac{b_{t}}{q_{t}}\right)\right)^{-\frac{1}{2}}\left(\sum_{n=t}^{r} 1\right)\right)^{-2} \\
& =M^{\prime}(k, r)\left(\sum_{s=1}^{k}\left(p_{s} \phi\left(\frac{a_{s}}{p_{s}}\right)\right)^{\frac{1}{3}}(k-s+1)\right)^{3} \\
& \times\left(\sum_{t=1}^{r}\left(q_{t} \psi\left(\frac{b_{t}}{q_{t}}\right)\right)^{-\frac{1}{2}}(r-t+1)\right)^{-2} \\
& =M^{\prime}(k, r)\left(\sum_{m=1}^{k}\left(p_{m} \phi\left(\frac{a_{m}}{p_{m}}\right)\right)^{\frac{1}{3}}(k-m+1)\right)^{3} \\
& \times\left(\sum_{n=1}^{r}\left(q_{n} \psi\left(\frac{b_{n}}{q_{n}}\right)\right)^{-\frac{1}{2}}(r-n+1)\right)^{-2} .
\end{aligned}
$$

The proof is complete.

Remark 2. Inequality (6) is just an inverse of the following Inequality B, which was proved by Pachpatte [6]: 
Inequality B.

$$
\begin{aligned}
\sum_{m=1}^{k} \sum_{n=1}^{r} \frac{\phi\left(A_{m}\right) \psi\left(B_{n}\right)}{m+n} \leq M(k, r)\left(\sum_{m=1}^{k}(k-m+1)\left(p_{m} \phi\left(\frac{a_{m}}{p_{m}}\right)\right)^{2}\right)^{\frac{1}{2}} \\
\times\left(\sum_{n=1}^{r}(r-n+1)\left(q_{n} \psi\left(\frac{b_{n}}{q_{n}}\right)\right)^{2}\right)^{\frac{1}{2}},
\end{aligned}
$$

where

$$
M(k, r)=\frac{1}{2}\left(\sum_{m=1}^{k}\left(\frac{\phi\left(P_{m}\right)}{P_{m}}\right)^{2}\right)^{\frac{1}{2}}\left(\sum_{n=1}^{r}\left(\frac{\psi\left(Q_{n}\right)}{Q_{n}}\right)^{2}\right)^{\frac{1}{2}} .
$$

Similarly, the following two theorems can also be established.

Theorem 3. Let $\left\{a_{m}\right\}$ and $\left\{b_{n}\right\}$ be as in Theorem 1 and set $A_{m}=(1 / m) \sum_{s=1}^{m} a_{s}$ and $B_{n}=(1 / n) \sum_{t=1}^{n} b_{t}$, for $m=1,2, \ldots, k$ and $n=1,2, \ldots, r$, where $k$ and $r$ are natural numbers. Let $\phi$ and $\psi$ be two real-valued, nonnegative, and concave functions defined on $\mathbb{R}_{+}$. Then for $3 n-2 m>0$,

$$
\begin{aligned}
\sum_{m=1}^{k} \sum_{n=1}^{r} \frac{m n}{3 n-2 m} \phi\left(A_{m}\right) \psi\left(B_{n}\right) \geq k^{-2} & r^{3}\left(\sum_{m=1}^{k}(k-m+1)\left(\phi\left(a_{m}\right)\right)^{\frac{1}{3}}\right)^{3} \\
& \times\left(\sum_{n=1}^{r}(r-n+1)\left(\psi\left(b_{n}\right)\right)^{-\frac{1}{2}}\right)^{-2} .
\end{aligned}
$$

Remark 3. Inequality (7) is just an inverse of the following Inequality $\mathrm{C}$, which was proved by Pachpatte [6]:

Inequality $\mathbf{C}$.

$$
\begin{aligned}
\sum_{m=1}^{k} \sum_{n=1}^{r} \frac{m n}{m+n} \phi\left(A_{m}\right) \psi\left(B_{n}\right) \leq \frac{1}{2}(k r)^{\frac{1}{2}}\left(\sum_{m=1}^{k}(k-m+1)\left(\phi\left(a_{m}\right)\right)^{2}\right)^{\frac{1}{2}} \\
\times\left(\sum_{n=1}^{r}(r-n+1)\left(\psi\left(b_{n}\right)\right)^{2}\right)^{\frac{1}{2}} .
\end{aligned}
$$

Theorem 4. Let $\left\{a_{m}\right\},\left\{b_{n}\right\},\left\{p_{m}\right\},\left\{q_{n}\right\}, P_{m}, Q_{n}$ be as in Theorem 2 and put $A_{m}=\left(1 / p_{m}\right) \sum_{s=1}^{m} p_{s} a_{s}, B_{n}=\left(1 / Q_{n}\right) \sum_{t=1}^{n} q_{t} b_{t}$, for $m=1,2, \ldots k, n=1,2, \ldots, r$, where $k$ and $r$ are natural numbers. Let $\phi$ and $\psi$ be as defined in Theorem 3. Then 
for $3 n-2 m>0$,

$$
\begin{aligned}
\sum_{m=1}^{k} \sum_{n=1}^{r} \frac{P_{m} Q_{n} \phi\left(A_{m}\right) \psi\left(B_{n}\right)}{3 n-2 m} \geq k^{-2} & r^{3}\left(\sum_{m=1}^{k}(k-m+1)\left(p_{m} \phi\left(a_{m}\right)\right)^{\frac{1}{3}}\right)^{3} \\
& \times\left(\sum_{n=1}^{r}(r-n+1)\left(q_{n} \psi\left(b_{n}\right)\right)^{-\frac{1}{2}}\right)^{-2} .
\end{aligned}
$$

Remark 4. Inequality (8) is just an inverse of the following Inequality D, which was proved by Pachpatte [6]:

\section{Inequality D.}

$$
\begin{aligned}
\sum_{m=1}^{k} \sum_{n=1}^{r} \frac{P_{m} Q_{n} \phi\left(A_{m}\right) \psi\left(B_{n}\right)}{m+n} \leq \frac{\sqrt{k r}}{2}\left(\sum_{m=1}^{k}(k-m+1)\left(p_{m} \phi\left(a_{m}\right)\right)^{2}\right)^{\frac{1}{2}} \\
\times\left(\sum_{n=1}^{r}(r-n+1)\left(q_{n} \psi\left(b_{n}\right)\right)^{2}\right)^{\frac{1}{2}}
\end{aligned}
$$

The proofs of Theorems 3 and 4 can be completed by following the same steps as in the proof of Theorem 2 with suitable changes. Here, we omit the details.

\section{REFERENCES}

[1] E. Beckenbach and R. Bellman, An Introduction to Inequalities, ser. New Mathematical Library. New York: Random House, 1961, vol. 3.

[2] M. Gao, "On Hilbert's inequality and its applications," J. Math. Anal. Appl., vol. 212, no. 1, pp. 316-323, 1997.

[3] M. Gao and B. Yang, "On the extended Hilbert's inequality," Proc. Amer. Math. Soc., vol. 126, no. 3, pp. 751-759, 1998.

[4] G. H. Hardy, J. E. Littlewood, and G. Pólya, Inequalities. Cambridge: Cambridge University Press, 1952, 2d ed.

[5] K. Jichang, "On new extensions of Hilbert's integral inequality," J. Math. Anal. Appl., vol. 235, no. 2, pp. 608-614, 1999.

[6] B. G. Pachpatte, "On some new inequalities similar to Hilbert's inequality," J. Math. Anal. Appl., vol. 226, no. 1, pp. 166-179, 1998.

[7] B. G. Pachpatte, "Inequalities similar to certain extensions of Hilbert's inequality," J. Math. Anal. Appl., vol. 243, no. 2, pp. 217-227, 2000.

[8] B. Yang, "On new generalizations of Hilbert's inequality," J. Math. Anal. Appl., vol. 248, no. 1, pp. 29-40, 2000.

[9] C.-j. Zhao, "Generalization on two new Hilbert type inequalities," J. Math. (Wuhan), vol. 20, no. 4, pp. 413-416, 2000.

[10] C.-J. Zhao and L. Debnath, "Some new inverse type Hilbert integral inequalities," J. Math. Anal. Appl., vol. 262, no. 1, pp. 411-418, 2001. 
Authors' addresses

Zhao Changjian

Department of Information and Mathematics Sciences, College of Science, China Jiliang University, Hangzhou 310018, P. R. China

E-mail address: chjzhao@163.com

Mihály Bencze

6 Harmanului St., 505600 Sacele-Négyfalu, Jud. Brasov, Romania 